\title{
Sinterização por micro-ondas de ferrita de níquel sintetizada pelo método Pechini
}

\section{(Microwave sintering of nickel ferrite synthesized by the Pechini method)}

\author{
J. R. Muñoz Hoyos ${ }^{1}$, F. L. Zabotto' ${ }^{2}$ D. Garcia', R. H. G. A. Kiminami ${ }^{1}$ \\ ${ }^{I}$ Departamento de Engenharia de Materiais, ${ }^{2}$ Departamento de Física \\ Universidade Federal de S. Carlos, Rod. Washington Luiz, km 235, S. Carlos, SP 13565-905 \\ ruth@ufscar.br
}

\begin{abstract}
Resumo
A sinterização por micro-ondas é uma técnica que oferece enorme potencial para a fabricação de diversos materiais cerâmicos com microestruturas cerâmicas diferenciadas. Este trabalho teve por objetivo sinterizar cerâmicas de ferrita de níquel $\left(\mathrm{NiFe}_{2} \mathrm{O}_{4}\right)$ em forno de micro-ondas, visando controle de crescimento de grão e alta densificação. Para realizar esta investigação, foi utilizado o processo de síntese por Pechini para a preparação do pó das ferritas de níquel. Amostras desse material foram sinterizadas em forno convencional e de micro-ondas. A caracterização foi feita por difração de raios X, microscopia eletrônica de varredura, determinação de densidade aparente e obtenção das curvas de histerese magnética. A sinterização da ferrita de níquel por micro-ondas levou apenas $10 \%$ do tempo gasto da sinterização convencional. As amostras apresentaram menor tamanho de grão, e densidade e magnetização de saturação semelhantes às obtidas pela sinterização convencional, mostrando o alto potencial de aplicação da técnica de microondas para o processamento desses materiais.
\end{abstract}

Palavras-chave: método Pechini, sinterização, micro-ondas, ferrita de níquel.

\begin{abstract}
Microwave sintering is a technique with a huge potential for the manufacture of a variety of ceramic materials with controlled ceramic microstructure. The objective of the present work was the microwave sintering of $\mathrm{NiFe}_{2} \mathrm{O}_{4}$ in a microwave oven. In this research, we used a $\mathrm{NiFe}_{2} \mathrm{O}_{4}$ synthesized by Pechini method. Samples of this powder were sintered by the conventional and microwave process, and characterized by X-ray diffraction and scanning electron microscopy, and their density and magnetic properties were also determined. The microwave sintering process took less than $10 \%$ of the time required for conventional sintering, and the samplebulk density and saturation magnetization values were similar to those observed forthe conventional sintered ones.
\end{abstract}

Keywords: Pechini method, microwave sintering, nickel ferrite.

\section{INTRODUÇÃO}

A ferrita de níquel $\left(\mathrm{NiFe}_{2} \mathrm{O}_{4}\right)$ é um material cerâmico que apresenta estrutura do tipo espinélio inversa [1]. É considerado como um material ferrimagnético mole, pois possuí baixo campo coercitivo e alta permeabilidade [2]. Além disso, entre outros materiais magnéticos, apresenta relativamente alta resistividade elétrica, dureza mecânica, estabilidade química e custos razoáveis, que permitem uma ampla variedade de aplicações em diversos dispositivos eletrônicos [3]. As propriedades magnéticas específicas desse tipo de material dependem do grau de densificação e da microestrutura do produto final que, por sua vez, se vinculam a fatores, tais como características do pó e parâmetros de sinterização $[4,5]$. Convencionalmente, os pós de $\mathrm{NiFe}_{2} \mathrm{O}_{4}$ são obtidos por reação em estado sólido de mistura de óxidos, mas grandes esforços têm sido realizados no desenvolvimento de métodos alternativos de síntese química visando o controle das características do pó, principalmente pureza, morfologia e tamanho médio das partículas. Na síntese especificamente de ferritas de níquel, vários métodos de síntese têm sido utilizados, entre eles, o citrato gel [6], coprecipitação [7, 8], reação por combustão [4, 9-11], Pechini [12], e sol-gel [8]. Entre os métodos de síntese mencionados, o método Pechini permite preparar pós com alta pureza, boa homogeneidade em escala atômica, e tamanho médio de partículas na escala nanométrica [12]. O método consiste na formação de quelatos entre cátions metálicos, em solução aquosa com um ácido hidrocarboxílico. A presença do poliálcool (etileno glicol) na solução permite promover uma polimerização entre o citrato do íon metálico e o etileno glicol. O aquecimento com temperaturas moderadas (100 ${ }^{\circ} \mathrm{C}$ ) causa reações de esterificação, dando origem a uma resina polimérica altamente viscosa $[12,13]$. O polímero formado apresenta grande homogeneidade na dispersão dos cátions metálicos e, após tratamento térmico adequado, a parte orgânica é eliminada, obtendo-se a fase desejada.

Independentemente do método de síntese, materiais cerâmicos à base de ferritas normalmente são sinterizados em forno convencional. Pesquisas em métodos alternativos 
de queima, visando o controle do crescimento dos grãos, têm despertado interesse especial na comunidade científica, pois se vislumbram propriedades diferenciadas para escalas menores do que as micrométricas. $\mathrm{O}$ aquecimento por microondas é um processo alternativo para sinterizar materiais cerâmicos com microestruturas diferenciadas. Isto porque, enquanto na queima convencional ocorre, inicialmente, o aquecimento da superfície do material e, só em seguida, por processos de convecção e condução, a energia térmica é transferida para o interior do corpo cerâmico, no aquecimento por micro-ondas a energia de micro-ondas é transformada em calor diretamente dentro do material (a partir da interação das moléculas e átomos com o campo eletromagnético, o que possibilita o aquecimento interno e volumétrico do material) [14-21]. Assim, o aquecimento por meio de micro-ondas é uma forma de processamento que, em virtude de características particulares, possibilita ultrapassar as limitações do processo de queima convencional. Essas características são oriundas dos mecanismos de geração de calor envolvidos na utilização das micro-ondas, que permitem um aquecimento volumétrico da peça, de forma rápida e mais uniforme que no caso da sinterização convencional [14]. Pelo fato de que, com a sinterização em elevada temperatura, em um curto período de tempo, produtos de maior ou igual densidade relativa e menor tamanho de grão podem ser obtidos, comparativamente à sinterização convencional [16]. Dessa forma, vários são os benefícios do uso da energia de micro-ondas na sinterização, tais como redução do tamanho médio dos grãos e no tempo de processamento, e economia de energia. A partir dessas motivações, este trabalho teve por objetivo investigar a aplicação de sinterização por micro-ondas de ferritas de níquel preparadas pelo método Pechini, e avaliar os aspectos microestruturais e as propriedades magnéticas das cerâmicas processadas.

\section{MATERIAIS E MÉTODOS}

Para a síntese da ferrita de níquel, $\mathrm{NiFe}_{2} \mathrm{O}_{4}$, inicialmente foi misturado o etilenoglicol com ácido cítrico em uma relação molar $4: 1$, em $70{ }^{\circ} \mathrm{C}$, até obter-se uma solução transparente. Simultaneamente, em $50 \mathrm{~mL}$ de água destilada foram diluídos o nitrato de níquel (Merck, 99 \% de pureza) e o nitrato de ferro (Merck, $99 \%$ de pureza) nas quantidades estequiométricas estabelecidas. Essas soluções foram adicionadas na de ácido cítrico e etilenoglicol e deixadas em agitação. A solução obtida foi aquecida em uma placa com temperatura entre 120 e $140{ }^{\circ} \mathrm{C}$, visando eliminar o solvente e formar uma resina polimérica, a qual se levou a um processo de pré-calcinação a $250{ }^{\circ} \mathrm{C}$, durante $24 \mathrm{~h}$, e por fim foi calcinada em um forno tipo mufla (EDG 3000) a $600{ }^{\circ} \mathrm{C}$, durante $1 \mathrm{~h}$. Os pós cerâmicos obtidos foram caracterizados por: difração de raios X (DRX), utilizando-se um difractômetro Rigaku, radiação $\mathrm{Cu} K \alpha$, área superficial (BET), com um equipamento Micromeritics, modelo Gemini - 2370; e por microscopia eletrônica de varredura, com um microscópio FEG Philips XL30.
Os pós foram conformados em pastilhas cilíndricas com diâmetro de $10 \mathrm{~mm}$ por prensagem isostática a $200 \mathrm{MPa}$ e a densidade a verde determinada. As amostras conformadas foram sinterizadas em forno de micro-ondas (Cober Electronics MS6K) a 2,45 GH z e 2,1 kW de potência, com material susceptor como agente auxiliar de aquecimento para a sinterização híbrida, com uma taxa de aquecimento de $100^{\circ} \mathrm{C} / \mathrm{min}$ de $1150{ }^{\circ} \mathrm{C}$ a $1250^{\circ} \mathrm{C}$ (ao ar), com patamares de 30 e $60 \mathrm{~min}$. A sinterização pelo método convencional foi em forno tubular Lindberg Blue $\mathrm{M}$, com taxas de aquecimento $5{ }^{\circ} \mathrm{C} / \mathrm{min}$ por $60 \mathrm{~min}$ a $1150{ }^{\circ} \mathrm{C}$ e $1250{ }^{\circ} \mathrm{C}$, com uma taxa de esfriamento de $5{ }^{\circ} \mathrm{C} / \mathrm{min}$ e tempo de duração do ciclo de sinterização de $\sim 11 \mathrm{~h}$. As medidas de densidade aparente (DA) das amostras sinterizadas foram pelo método de imersão pelo princípio de Arquimedes (norma ABNT-NBR6620). As caracterizações físicas e microestruturais foram feitas por difração de raios X (difratômetro Siemens D-5005) e microscopia eletrônica de varredura (Philips XL-30 FEG). As amostras submetidas à caracterização pelo MEV foram fraturadas e o tamanho médio de grão foi medido pela técnica linear dos interceptos. Para cada região, foram contados no mínimo 150 grãos, utilizando-se o software Image Pro Plus. As amostras foram caracterizadas magneticamente a partir da obtenção das curvas de magnetização vs. campo magnético, à temperatura ambiente, num sistema de medidas PPMS Quantum Design 7100.

\section{RESULTADOS E DISCUSSÃO}

Inicialmente, foi feita a caracterização dos pós de $\mathrm{NiFe}_{2} \mathrm{O}_{4}$ sintetizados pelo método Pechini. Eles apresentaram área superficial $11,3 \mathrm{~m}^{2} \mathrm{~g}_{-}{ }^{1}$ e tamanho de partícula $99 \mathrm{~nm}$, calculado a partir dos resultados da área superficial [19]. A Fig. 1 apresenta os resultados de difração de raios $\mathrm{X}$ do pó calcinado. Observam-se, além da fase cristalina da ferrita de níquel, traços de hematita $\left(\mathrm{Fe}_{2} \mathrm{O}_{3}\right)$. A partir da largura dos picos obtidos por DRX, foi calculado o tamanho médio de cristalito pelo método de Scherrer. O valor obtido foi 77 $\mathrm{nm}$, inferior ao calculado a partir da área superficial. Essa diferença pode ser atribuída ao modelo utilizado no cálculo do tamanho médio a partir dos valores da área superficial, no qual se consideram idealmente partículas com o formato esférico.

A Fig. 2 apresenta o resultado obtido por microscopia eletrônica de varredura do pó calcinado de ferrita de níquel. Observa-se uniformidade tanto no tamanho e formato das partículas, como nos tamanhos inferiores a $100 \mathrm{~nm}$, corroborando os resultados discutidos anteriormente.

Na Tabela I estão apresentados os resultados para a densidade aparente relativa dos corpos cerâmicos verdes e sinterizados. Pode-se observar que a sinterização em forno de micro-ondas (SM1, SM2) permitiu obter cerâmicas com valores de densidade comparáveis às obtidas na sinterização convencional (SC). Uma vantagem desse resultado é que no forno de micro-ondas foi usada uma taxa de sinterização 20 vezes maior do que a usada no forno convencional, o que 


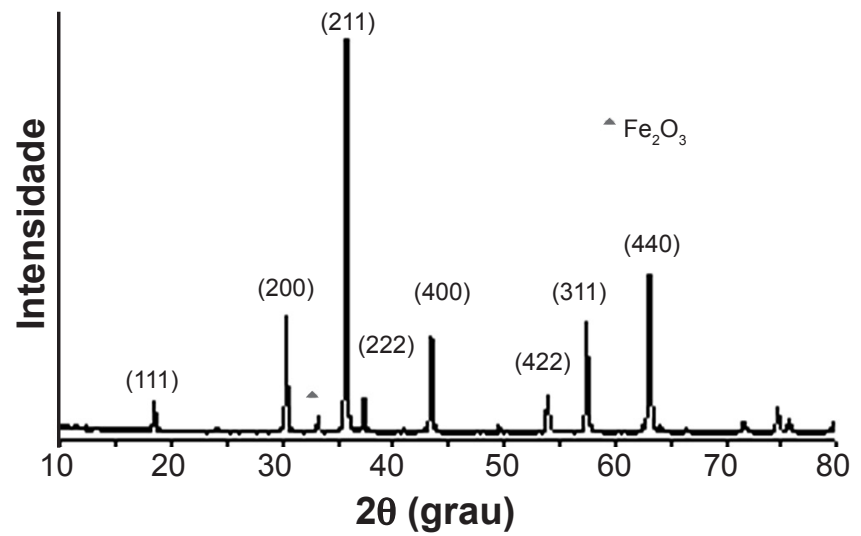

Figura 1: Perfil de difração de raios $\mathrm{X}$ do pó de ferrita de níquel sintetizado pelo método Pechini, calcinado a $600{ }^{\circ} \mathrm{C}$. A indexação (hkl) refere-se aos arquivos JCPDS 86-2267 e JCPDS 72-469, das fases $\mathrm{NiFe}_{2} \mathrm{O}_{4}$ e $\mathrm{Fe}_{2} \mathrm{O}_{3}$, respectivamente.

[Figure 1: X-ray powder diffraction pattern of nickel ferrite synthetized by the Pechini method. The (hkl) indexing refers to JCPDS file 86-2267 and JCPDS file 72-469, NiFe $\mathrm{O}_{4}$ and $\mathrm{Fe}_{2} \mathrm{O}_{3}$ phases, respectively.]

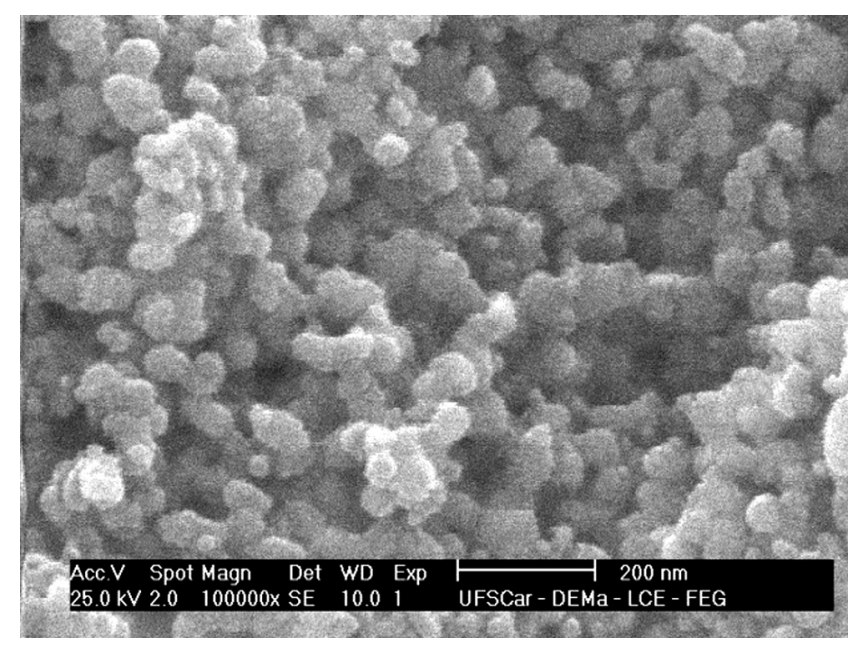

Figura 2: Micrografia obtida por MEV do pó da ferrita de níquel sintetizado pelo método Pechini e calcinado a $600{ }^{\circ} \mathrm{C}$.

[Figure 2: SEM micrograph of nickel ferrite powder synthesized by the Pechini method and calcined at $600^{\circ} \mathrm{C}$.]

permitiu uma redução considerável no tempo de queima, pois de $9 \mathrm{~h}$ para o caso da (convencional), utilizaram-se, no máximo, $2 \mathrm{~h}$ (dependendo do tempo de patamar utilizado) no caso de (micro-ondas). Observou-se também que, com o aumento do tempo de sinterização de 30 (SM1) para 60 min (SM2), a $1150^{\circ} \mathrm{C}$, não houve aumento na densidade das amostras.

AFig. 3 apresenta as micrografias de fratura das cerâmicas sinterizadas em forno de micro-ondas (SM1 e SM2) e convencional (SC). É possível observar que as amostras sinterizadas por micro-ondas (SM1 e SM2) apresentaram uma estrutura uniforme, com tamanhos médios de grãos um pouco inferiores ao tamanho obtido nas amostras sinterizadas em forno convencional (SC). O tamanho médio de grãos das amostras sinterizadas em forno convencional (SC) foi 3,9 $\pm 0,9 \mu \mathrm{m}$ e das amostras sinterizadas em forno de micro-ondas, foi 1,8 $\pm 0,6$ e 2,6 $\pm 0,8 \mu \mathrm{m}$, para SM1 e SM2, respectivamente. Ou seja, mesmo para períodos totais de queima bem menores, a sinterização por microondas permitiu obter microestruturas com tamanho médio de grãos, embora um pouco menores, na mesma ordem de grandeza daquelas obtidas no forno convencional. Provavelmente a elevada taxa de aquecimento inibiu parcialmente o crescimento de grãos, pelo menor período (comparativamente ao do caso convencional) que o material fica em temperaturas inferiores a do patamar. Também pode ser observado que o aumento do tempo de patamar de 30 para $60 \mathrm{~min}$ no forno de micro-ondas, resultou em um aumento de $\sim 50 \%$ do tamanho médio de grãos, podendose concluir que, no caso da queima por micro-ondas, é realmente durante o tratamento a alta temperatura que o crescimento de grão ocorre.

A Fig. 4 e a Tabela II apresentam os resultados de algumas propriedades magnéticas, das amostras de ferrita de níquel SM1, SM2 e SC, determinados a partir das medidas do ciclo de histerese para cada condição de sinterização. Dos dados da Tabela II, pode-se observar que a sinterização por micro-ondas permitiu a obtenção de amostras com valores de magnetização de saturação $M s$ muito semelhantes aos da sinterização convencional. Esses valores de magnetização, à temperatura ambiente, estão de acordo com os reportados [22], $M s=45 \mathrm{emu} / \mathrm{g}$, em amostras de mesma composição sintetizadas por mistura de óxidos e sinterizadas em forno convencional, e superiores aos reportados [9] em amostras sintetizadas por combustão e sinterizadas em forno convencional, $M s=32 \mathrm{emu} / \mathrm{g}$. Os valores da magnetização remanente, por sua vez, tanto nas amostras sinterizadas

Tabela I - Valores para a densidade aparente relativa dos corpos cerâmicos de ferrita de níquel a verde, $\rho_{\mathrm{v}}$, e sinterizados, $\rho_{\mathrm{ap}}$, para a sinterização convencional e por micro-ondas, com as respectivas condições de queima (taxa de aquecimento, e tempo e temperatura de patamar).

[Table I - Relative density values for the nickel ferrite ceramic bodies before $\left(\rho_{V}\right)$ and after conventional and microwave sintering $\left(\rho_{a p}\right)$ and related fired conditions (heating rate, and soaking time and temperature).]

\begin{tabular}{ccccc}
\hline Sinterização & $\begin{array}{c}\text { Taxa de aquecimento } \\
\left({ }^{\circ} \mathrm{C} / \mathrm{min}\right)\end{array}$ & $\begin{array}{c}\text { Temperatura e tempo } \\
\text { de patamar }\end{array}$ & $\begin{array}{c}\rho_{\mathrm{v}} \\
(\%)\end{array}$ & $\begin{array}{c}\rho_{\mathrm{ap}} \\
(\%)\end{array}$ \\
\hline $\mathrm{SC}$ & 5 & $1150{ }^{\circ} \mathrm{C} / 60 \mathrm{~min}$ & $51,0 \pm 0,4$ & $93,5 \pm 0,5$ \\
$\mathrm{SM} 1$ & 100 & $1150{ }^{\circ} \mathrm{C} / 30 \mathrm{~min}$ & $51,0 \pm 0,4$ & $91,9 \pm 0,2$ \\
$\mathrm{SM} 2$ & 100 & $1150{ }^{\circ} \mathrm{C} / 60 \mathrm{~min}$ & $51,0 \pm 0,4$ & $92,0 \pm 0,1$ \\
\hline
\end{tabular}



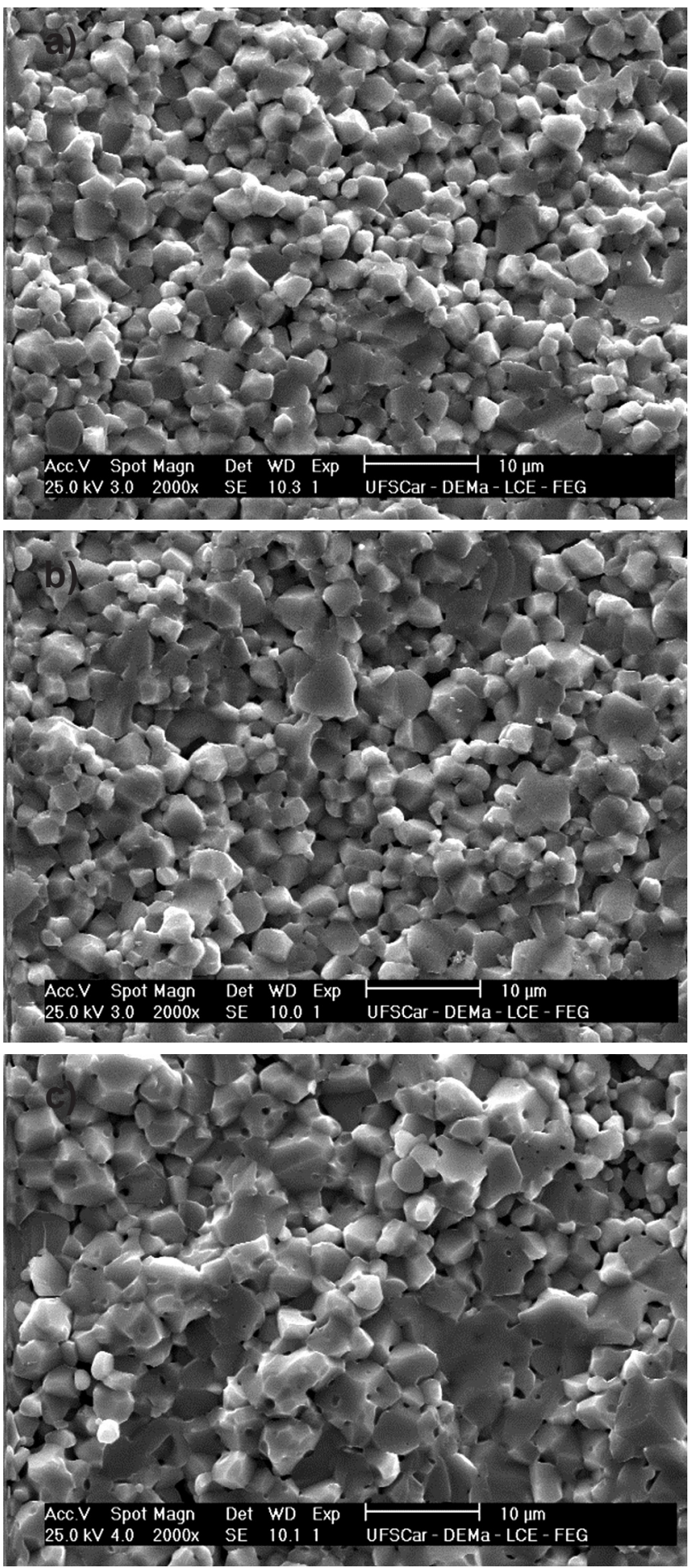

Figura 3: Micrografias obtidas por microscopia eletrônica de varredura de ferrita de níquel sinterizada por micro-ondas, a) SM1 e b) SM2, e convencionalmente, c) SC.

[Figure 3: SEM micrographs of nickel ferrite ceramics sintered by microwave, a) SM1 and b) SM2, and by the conventional method, c) SC.]

em forno convencional como em micro-ondas, foram bem inferiores a $1 \mathrm{emu} / \mathrm{g}$ e aos reportados pelos mesmos autores acima citados, de $\mathrm{Mr}=19,70 \mathrm{emu} / \mathrm{g}$ [9] e $\mathrm{Mr}=3,46$ emu/g [22]. Observou-se que com o aumento do tempo de
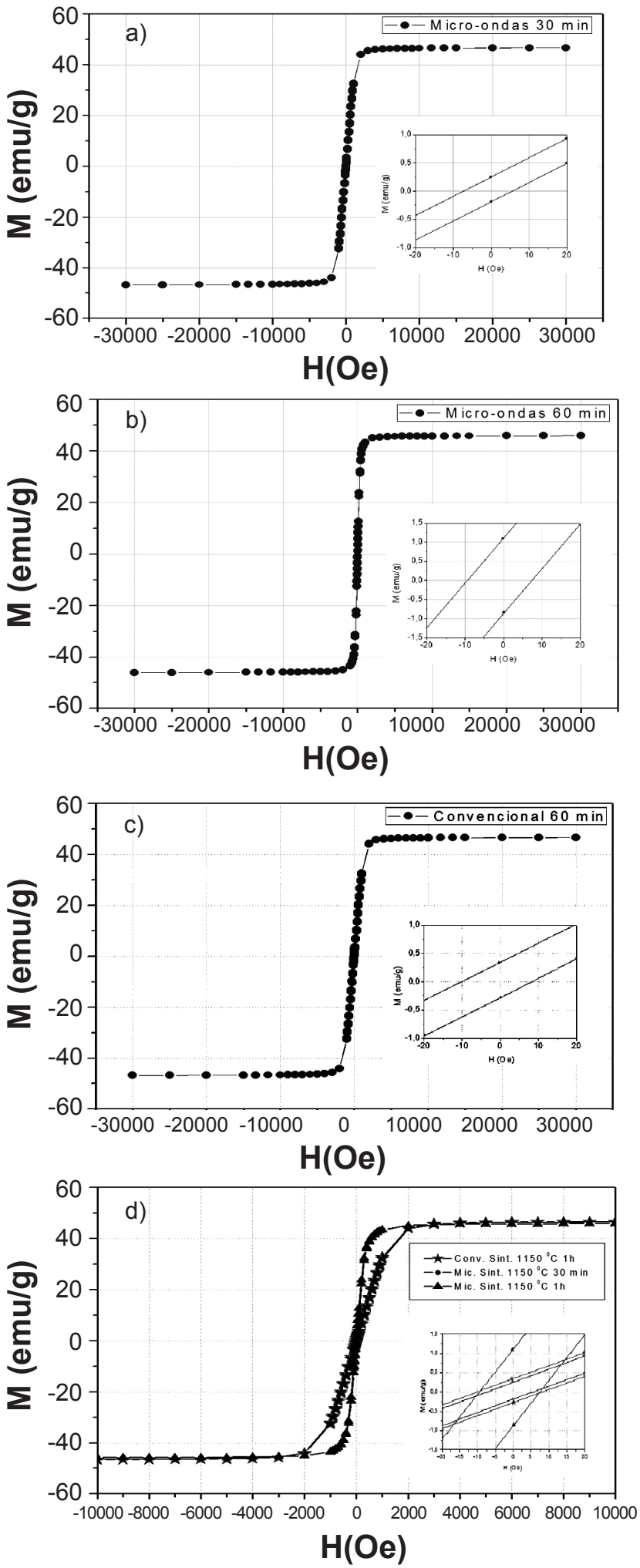

Figura 4: Curvas de histerese magnética das amostras sinterizadas por micro-ondas, a) SM1 e b) SM2, e convencionalmente, c) SC; e, d), a superposição de SM1, SM2 e SC. Em destaque, em cada gráfico, a região no entorno de campo magnético nulo foi ampliada para melhor visualização e comparação dos resultados da magnetização remanente e do campo coercitivo.

[Figure 4: Magnetic hysteresis loops of samples sintered by microwave, a) SM1 and b) SM2, and by the conventional method, c) $S C$; and d) overlay of SM1, SM2, and SC.] 
sinterização por micro-ondas, de 30 a 60 min (SM1 e SM2), houve um aumento significativo do valor de $\mathrm{Mr}$, que passou de 0,22 a $0,98 \mathrm{emu} / \mathrm{g}$. Esse resultado pode ser mais bem observado na Fig. 4(d), na qual os ciclos de histerese estão superpostos. Tanto as amostras sinterizadas por micro-ondas como as sinterizadas em forno convencional apresentaram valores relativamente baixos de campo coercitivo, o que é característico de materiais ferrimagnéticos moles. Nas amostras SM1 e SM2 o valor do campo coercitivo foi inferior, quando comparados com as amostras SC. Assim, é possível que estes valores de $M r$ e de $H c$ possam estar diretamente relacionados com o tamanho médio de grãos, e que microestruturas mais finas, que é o caso, resultem em magnetização remanente e campo coercitivo menores.

Tabela II - Tamanho médio de grãos e propriedades magnéticas das cerâmicas de ferrita de níquel obtidas a partir dos ciclos de histerese, onde Ms é a magnetização de saturação, Mr, a magnetização remanente, e, Hc, o campo coercitivo.

[Table II - Average grain size and magnetic properties of the nickel ferrite ceramics obtained from the hysteresis loops. Ms (saturation magnetization), Mr (remanent magnetization) and Hc (coercive field).]

\begin{tabular}{ccccc}
\hline Sinterização & $\begin{array}{c}\text { Tamanho } \\
\text { médio de } \\
\text { grãos } \\
(\mu \mathrm{m})\end{array}$ & $\begin{array}{c}M_{s} \\
(\mathrm{emu} / \mathrm{g})\end{array}$ & $\begin{array}{c}M_{r} \\
(\mathrm{emu} / \mathrm{g})\end{array}$ & $\begin{array}{c}H_{c} \\
(\mathrm{Oe})\end{array}$ \\
\hline SC & $3,9 \pm 0,9$ & 46,50 & 0,32 & 9,20 \\
$\mathrm{SM} 1$ & $1,8 \pm 0,6$ & 46,40 & 0,22 & 6,54 \\
$\mathrm{SM} 2$ & $2,6 \pm 0,8$ & 45,81 & 0,98 & 8,42 \\
\hline
\end{tabular}

\section{CONCLUSÕES}

O método de síntese Pechini permitiu obter pós de ferrita de níquel cristalinos e presença de traços de hematita após calcinação de $600{ }^{\circ} \mathrm{C}$, eliminada após sinterização, tanto por micro-ondas quanto convencionalmente, e tamanho médio de partículas inferiores a $100 \mathrm{~nm}$. Com a sinterização por micro-ondas e pelo método convencional, foram obtidas amostras de ferritas de níquel com valores de densidade relativa acima de $90 \%$, e magnetização de saturação, semelhantes aos reportados na literatura. Os baixos valores da magnetização remanente e do campo coercitivo estão associados com o tamanho médio de grão, tanto na sinterização por micro-ondas como convencional. A sinterização por micro-ondas, além de permitir a obtenção de uma microestrutura homogênea com tamanhos de grãos inferiores aos obtidos por sinterização convencional e aos reportados na literatura, reduz o tempo total de queima no micro-ondas em até $90 \%$.

\section{AGRADECIMENTOS}

Os autores agradecem ao CNPq, CAPES e FAPESP pelas bolsas e financiamento na pesquisa. Agradecem ao Prof. Dr. Adilson J. A. de Oliveira e ao Mestre Alexandre J. Gualdi, do Grupo de Supercondutividade e Magnetismo, GSM, do Departamento de Física da Universidade Federal de S. Carlos, pela caracterização magnética.

\section{REFERÊNCIAS}

[1] H. Perron, T. Mellier, C. Domain, J. Roques, E. Simoni, R. Drotand, H Catalette, J. Phys. Condens. Matter. 19 (2007) 10.

[2] R. B. Pujar, S. N. Kulkarni, C. B. Bellad, B. K. Chougule, J. Mater. Sci. Lett. 16 (1997) 1668-1669.

[3] A. K. M. Akther Hossain, S. T. Mahmud, M. Seki, T. Kawai, H. Tabata, J. Mag. Mag. Mat. 312 (2007) 210-219.

[4] B. Domenichini, T. Caillot, Acta Mat. 51 (2003) 48154821.

[5] A. C. F. M. Costa, K. M. S. Vaina, E. J. Miola, S. G. Antônio, C. O. Paiva-Santos, R. H. G. A. Kiminami, Rev. Elet. Mater. Proc. 6.1 (2011) 06-10.

[6] S. Prasad, N. S. Gajbhiye, J. All. Comp. 265 (1998) $87-$ 92.

[7] K. Maaz, S. Karim, A. Mumtaz, S. K. Hasanain, J. Liu, J. L. Duan, J. Mag. Mag. Mat. 321 (2009) 1838-1842.

[8] M. Li, C. Liu, X. Gao, Adv. Mat. Res. 79-82 (2009) $305-$ 308.

[9] A. C. F. M. Costa, L. Gama, M. R. Morelli, R. H. G. A. Kiminami, Mater. Sci. For. 498-499 (2005) 618-623.

[10] A. C. F. M. Costa, M. R. Morelli, R. H. G. A. Kiminami, J. Mater. Sci. 5, 39 (2004) 1773-1778.

[11] A. C. F. M. Costa, A. M. D. Leite, H. S. Ferreira, R. H. G. A. Kiminami, J. Eur. Ceram. Soc. 10, 28 (2008) 2033 2037.

[12] A. N. Simões, D. A. Vieira, M. C. Silva, L. Gamma, A. C. F. M. Costa, R. H. G. A. Kiminami, Rev. Elet. Mater. Proc. 4.2 (2009) 26-31.

[13] M. P. Pechini, U.S: Patent, 3, 231, pp. 218, January 25 (1966).

[14] P. M. Souto, R. R. Menezes, R. H. G. A. Kiminami, J. Mater. Process. Technol. 209 (2009) 548-553.

[15] R. R. Menezes, P. M. Souto, R. H. G. A. Kiminami, Cerâmica 53, 325 (2007) 1-10.

[16] R. R. Menezes, P. M. Souto, R. H. G. A. Kiminami, Microwave Fast Sintering of Ceramic Materials in Sintering of Ceramics - New Emerging Techniques, In Tech (2012) p.3.

[17] A. C. Metaxas, J. G. P. Binner, In Advanced Ceramic Processing Technology, Ed. J. G. P. Binner, Noyes Publications, New Jersey, EUA (1990) 285.

[18] M. A. Janney, H. D. Kimeey, In Microwave Processing of Materials II, Materials Research Society Symposium Proceedings, Vol. 189, Ed. W. B. Snyder, Jr, W. H. Sutton, M. F. Iskander, D. L. Johnson, Mater. Res. Soc., Pittsburgh, PA (1991) 215.

[19] L. M. Sheppard, Am. Ceram. Soc. Bull. 67, 10 (1988) 1656-1661.

[20] J. D. Katz, R. D. Blake, Am. Ceram. Soc. Bull. 70, 8 
(1991) 1304-1308.

[21] J. S. Read, Principles of Ceramics Processing, $2^{\text {nd }}$ Ed., Wiley \& Sons, EUA (1995).
[22] G. Dixit, J. P. Singh, R. C. Srivastava, H. M. Agrawal, R. J. Choudhary, A. Gupta, Indian J. Pure Appl. Phys. 48 (2010) 287-291.

(Rec. 10/08/2012, Ac. 28/12/2012) 